\title{
Light-Induced Gaps in Semiconductor Band-to-Band Transitions
}

\author{
Q. T. Vu and H. Haug \\ Institut für Theoretische Physik, Universität Frankfurt, 60054 Frankfurt am Main, Germany
}

O. D. Mücke, T. Tritschler, and M. Wegener

Institut für Angewandte Physik, Universität Karlsruhe (TH), Wolfgang-Gaede-Straße 1, 76131 Karlsruhe, Germany

G. Khitrova and H. M. Gibbs

Optical Sciences Center, University of Arizona, Tucson, Arizona 85721, USA

(Received 4 December 2003; published 27 May 2004)

\begin{abstract}
We observe a triplet around the third harmonic of the semiconductor band gap when exciting $50-100 \mathrm{~nm}$ thin GaAs films with $5 \mathrm{fs}$ pulses at $3 \times 10^{12} \mathrm{~W} / \mathrm{cm}^{2}$. The comparison with solutions of the semiconductor Bloch equations allows us to interpret the observed peak structure as being due to a twoband Mollow triplet. This triplet in the optical spectrum is a result of light-induced gaps in the band structure, which arise from coherent band mixing. The theory is formulated for full tight-binding bands and uses no rotating-wave approximation.
\end{abstract}

DOI: 10.1103/PhysRevLett.92.217403

When an intense light field at frequency $\omega_{0}$ excites band-to-band transitions in a semiconductor, additional energy gaps can evolve within the original bands [Fig. 1(b)]. These so-called light-induced gaps [1-5] are the analog of the induced doublets, split by the Rabi frequency $\Omega_{\mathrm{R}}$, in a resonantly excited two-level system [Fig. 1(a)]. Here, two out of the four possible optical transitions are energetically degenerate. Hence, the optical spectrum consists of three peaks - the famous Mollow triplet for atoms [6,7] or excitons [8,9]. In the semiconductor band case, the light-induced gap in, e.g., the conduction band arises because the original conduction band and the one-photon sideband of the valence band lead to an avoided crossing. The corresponding Hopfield coefficients [1] determine the amount of conduction band admixture. It is crucial to note that this admixture can be finite even far away from the fictitious crossing point. This statement becomes particularly important for Rabi energies $\hbar \Omega_{\mathrm{R}}$ approaching the photon energy $\hbar \omega_{0}$, while it can be neglected for small Rabi energies. The latter case is tacitly assumed in previous graphical representations of calculated light-induced gaps. Importantly, as a result of this finite admixture, optical transitions between the induced bands are possible throughout an appreciable fraction of momentum space. Consequently, the transitions acquire considerable spectral weight. Again, two out of the four sets of possible optical transitions are energetically degenerate and a triplet results, which we will refer to as the two-band Mollow triplet in what follows.

Light-induced gaps in semiconductor bands have not unambiguously been observed yet. In order to observe the splitting, it clearly has to be larger than the damping. As typical electron dephasing times under relevant conditions are on the order of $10 \mathrm{fs}$ or less, the Rabi oscillation period has to be shorter than this value. This brings one
PACS numbers: 78.20.Bh, 42.50.Md, 42.65.Re, 78.47.+p

close to Rabi frequencies approaching the frequency of light - a regime which has previously been referred to as carrier-wave Rabi flopping [10]. In this regime, a splitting in the third-harmonic spectra has been observed [11]. However, as the measurements have been compared only with calculations on the basis of two-level systems [11], skepticism has been expressed whether this interpretation was correct indeed. Furthermore, later theoretical work [12] showed that the original experimental data (a)

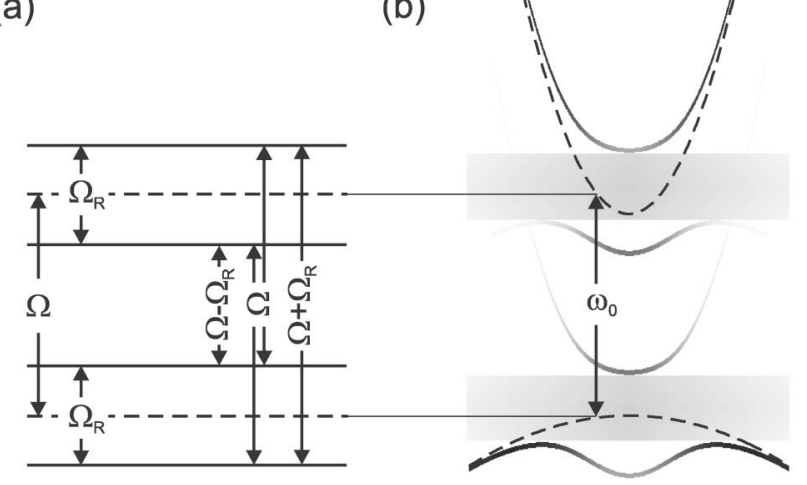

FIG. 1. (a) Scheme of the two-level system Mollow triplet for resonant excitation, i.e., $\Omega=\omega_{0}$. (b) Light-induced gaps (gray areas) are the analog for excitation within the bands of a semiconductor. The square modulus of the Hopfield coefficients [1] of the four resulting bands is superimposed onto them by a gray scale: Black corresponds to 1 , white to 0 . GaAs parameters are used and the Rabi energy $\hbar \Omega_{\mathrm{R}}$, the product of dipole matrix element and peak laser electric field, is chosen as $\hbar \Omega_{\mathrm{R}} / E_{\mathrm{g}}=$ 0.5 , with the band gap energy $E_{\mathrm{g}}=1.42 \mathrm{eV}, \hbar \omega_{0} / E_{\mathrm{g}}=1.1$. The dashed curves show the original conduction and valence band, respectively. Both (a) and (b) assume monochromatic excitation as well as negligible damping and are based on the rotating-wave approximation. 
were to some extent obscured by propagation effects within the sample.

In this Letter we present fully dynamical solutions of the semiconductor Bloch equations for tight-binding bands with semiphenomenological density- and energydependent dephasing and relaxation and without employing the rotating-wave approximation. Using parameters of the model semiconductor GaAs, we find that the lightinduced gaps in the original bands lead to a backgroundfree replica of the two-band Mollow triplet around the third harmonic of the fundamental band gap. The computed emission spectra are in almost quantitative agreement with corresponding thin-film measurements. This direct comparison of experiment and theory delivers the first unambiguous observation of light-induced gaps in semiconductor band-to-band transitions.

In our experiments, we employ 5 fs linearly polarized optical pulses from a Ti:sapphire mode-locked laser oscillator [13]. The pulses are tightly focused onto the GaAs film, held at room temperature, by means of a high numerical aperture $(\mathrm{NA}=0.5)$ reflective microscope objective. This leads to a spot radius of about $1 \mu \mathrm{m}$. We estimate that an average laser power of $36.5 \mathrm{~mW}$ in front of the sample at $f_{\mathrm{r}}=81 \mathrm{MHz}$ repetition frequency corresponds to a peak intensity of $I=2.8 \times 10^{12} \mathrm{~W} / \mathrm{cm}^{2}$. With dielectric constant $\epsilon=10.9$ for GaAs, we further estimate a peak electric field of $\tilde{E}_{0}=2.1 \times 10^{9} \mathrm{~V} / \mathrm{m}$ inside the sample. To get to these large fields in the experiment without damaging the sample, we have already chopped the laser intensity at about $100 \mathrm{~Hz}$ frequency and with about $3 \%$ duty cycle. The light emitted by the sample into the forward direction is collected by a second reflective microscope objective $(\mathrm{NA}=0.5)$, spectrally prefiltered by a sequence of four fused-silica Brewsterangle prisms to suppress the overwhelmingly prominent transmitted laser spectrum, and is sent into a grating spectrometer connected to a liquid-nitrogen cooled, back-illuminated, UV-enhanced charge-coupled device camera. The GaAs layers of thickness $l$ (without AlGaAs barriers) are grown directly on sapphire substrate in a molecular-beam epitaxy machine. The growth of the thin GaAs layer on the $0.43 \mathrm{~mm}$ thick, $51 \mathrm{~mm}$ diameter epiready sapphire substrate (University Wafer) mounted in an In-free molyblock was performed the same as for a GaAs wafer with a growth rate of $0.25 \mathrm{~nm} / \mathrm{s}$. We have investigated four samples with GaAs film thicknesses of $l=25 \mathrm{~nm}$ (two samples), $l=$ $50 \mathrm{~nm}$, and $l=100 \mathrm{~nm}$. Only the latter two samples turn out to have a damage threshold sufficiently large to actually perform the experiments at large Rabi energies. Two representative spectra for low and high excitation, respectively, are shown in Fig. 2. The spectra consist of the third harmonic and surface second-harmonic generation (SHG). This interpretation has been confirmed by additional experiments (not shown) in which we have varied the sample thickness $l$. At low excitation (lower solid curve), the third-harmonic spectrum consists of a

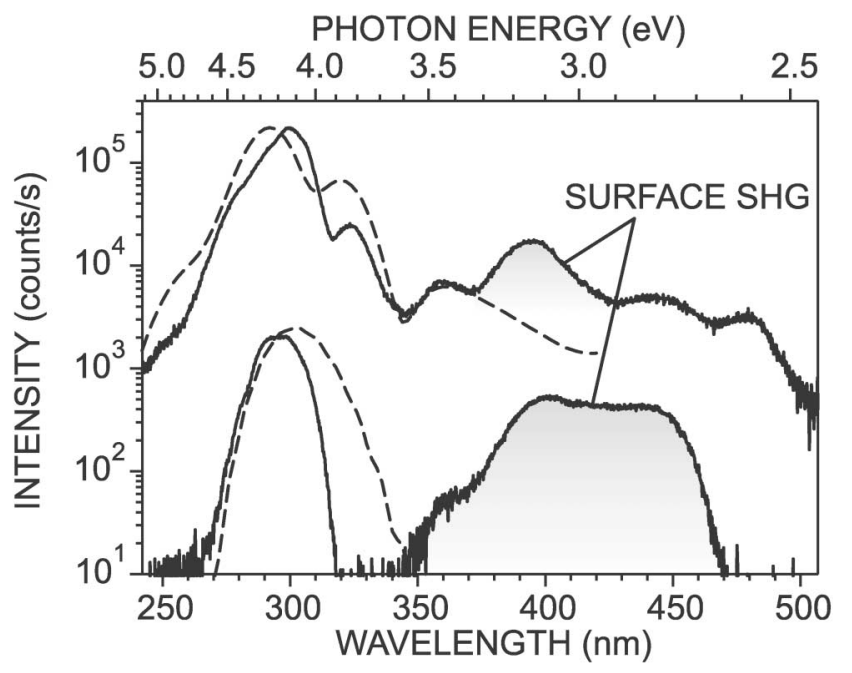

FIG. 2. Experiment (solid curves): Spectra of light emitted into the forward direction by a $l=100 \mathrm{~nm}$ thin GaAs film on sapphire substrate excited by 5 fs pulses. Lower curve: $I=$ $0.24 \times 10^{12} \mathrm{~W} / \mathrm{cm}^{2}$; upper curve: $I=2.8 \times 10^{12} \mathrm{~W} / \mathrm{cm}^{2}$ (same scale). We estimate that the latter translates into a peak electric field inside the GaAs film of $\tilde{E}_{0}=2.1 \times 10^{9} \mathrm{~V} / \mathrm{m}$. Theory (dashed curves): Corresponding result from the semiconductor Bloch equations for high $\left(\tilde{E}_{0}=1.65 \times 10^{9} \mathrm{~V} / \mathrm{m}\right)$ and low $\left(\tilde{E}_{0}=0.23 \times 10^{9} \mathrm{~V} / \mathrm{m}\right)$ excitation, respectively.

single peak around the third harmonic of the GaAs band gap $\left(3 E_{\mathrm{g}}=4.26 \mathrm{eV}\right)$. At high excitation (upper solid curve), this peak splits into three, one of which merges with the surface SHG. The following theoretical discussion will allow us to assign these three peaks to the twoband Mollow triplet arising from the light-induced gaps in the original semiconductor bands.

For the theoretical analysis we use the semiconductor Bloch equations. It is known $[3,4]$ that they are of the form of the Gross-Pitaevskii equation for the order parameter of a weakly nonideal Bose condensate. Because of the large spectral width of the pulses we do not make the usual effective mass approximation, but rather account for full bands. For simplicity, the (heavy-hole) valence (v) and conduction (c) bands are described by cubic tight-binding cosine bands. The coefficients are determined by fitting the resulting band structure to the GaAs band gap of $E_{\mathrm{g}}=1.42 \mathrm{eV}$ and its effective masses. The light-hole valence band leads to a smaller transition strength and is neglected. Furthermore, the Hartree-Fock (HF) Coulomb exchange interaction is taken into account,

$$
H_{\text {coul }}=-\sum_{\substack{n, m=c, \mathrm{c} \\ \vec{k}, \vec{q}}} V_{\vec{q}}\left(\rho_{n m, \vec{k}+\vec{q}}-\delta_{n, \mathrm{v}} \delta_{m, \mathrm{v}}\right) a_{n, \vec{k}}^{+} a_{m, \vec{k}},
$$

with the bare Coulomb potential $V_{\vec{q}}=$ $\left(v e^{2} / 4 \pi V \epsilon_{b}\right) \sum_{\vec{R} \neq 0}\left(e^{\mathrm{i} \vec{q} \vec{R}} /|\vec{R}|\right)$, where $\epsilon_{b}$ is the background dielectric constant, $v$ is the volume of a unit cell, $V$ is the crystal volume, and $\vec{R}$ is a lattice vector. Note that this crystal sum is generally different from the Fourier transform of $V(r)$. The density matrix $\rho$ is defined as $\rho_{n m, \vec{k}}=$ $\left\langle a_{m, \vec{k}}^{+} a_{n, \vec{k}}\right\rangle$. In (1), we have subtracted the exchange energy 
for the filled valence band, because that has already been accounted for in the band structure calculation. The coupling to the light field is treated in the usual dipole approximation [14]. We split the time derivative of the density matrix into a coherent part and a dissipative part due to various types of collisions,

$$
\frac{\partial}{\partial t} \rho_{n m, \vec{k}}(t)=\left.\frac{\partial}{\partial t} \rho_{n m, \vec{k}}(t)\right|_{\text {coh }}+\left.\frac{\partial}{\partial t} \rho_{n m, \vec{k}}(t)\right|_{\text {coll }} .
$$

The equation of motion of the coherent time evolution is

$$
\begin{aligned}
\left.\frac{\partial}{\partial t} \rho_{n m, \vec{k}}(t)\right|_{\text {coh }}= & -\frac{\mathrm{i}}{\hbar}\left(e_{n, \vec{k}}(t)-e_{m, \vec{k}}(t)\right) \rho_{n m, \vec{k}}(t) \\
& +\mathrm{i} \sum_{n_{1}}\left(\Omega_{n n_{1}, \vec{k}}^{R}(t) \rho_{n_{1} m, \vec{k}}(t)\right. \\
& \left.\quad-\rho_{n n_{1}, \vec{k}}(t) \Omega_{n_{1} m, \vec{k}}^{\mathrm{R}}(t)\right) .
\end{aligned}
$$

The HF renormalized energies are

$$
e_{n, \vec{k}}(t)=\epsilon_{n, \vec{k}}-\sum_{\vec{k}^{\prime}} V_{\vec{k}-\vec{k}^{\prime}}\left(\rho_{n n, \vec{k}^{\prime}}(t)-\delta_{n, \mathrm{v}}\right),
$$

with the single-particle energy bands $\epsilon_{n, \vec{k}}$ and the HF renormalized Rabi energies are

$$
\hbar \Omega_{n m, \vec{k}}^{\mathrm{R}}(t)=\left[d_{\vec{k}} E(t)+\sum_{\vec{q}} V_{\vec{q}} \rho_{n m, \vec{k}-\vec{q}}(t)\right] \sigma_{n m}^{x},
$$

where $d_{\vec{k}}$ is the momentum-dependent dipole matrix element, $E(t)$ the electric field of the laser pulse, and

$$
\sigma^{x}=\left(\begin{array}{ll}
0 & 1 \\
1 & 0
\end{array}\right)
$$

is a Pauli spin matrix. Note that the rotating-wave approximation has not been made. As we do not intend to treat the effects of different polarizations of light and momentum anisotropies, we introduce an isotropic density matrix which depends only on energy by a directional averaging.

In the regime of a few femtoseconds, the dissipative kinetics is governed by LO-phonon and by Coulomb scattering. The latter dominates at carrier densities of $10^{20} / \mathrm{cm}^{3}$ in the present experiments. A full quantum kinetic treatment along the lines of Ref. [15] is numerically too demanding under these conditions. Hence, we employ a phenomenological description - with parameters based on earlier findings [15,16]. For the dephasing of the off-diagonal density matrix elements we take into account that the damping is the sum of a constant contribution due to LO-phonon scattering and a term proportional to the third root of the carrier density $n$ [16]. To account for the additional energy dependence, we use the following dephasing for the various interband polarization $(p)$ components $(n \neq m)$,

$$
\left.\frac{\partial}{\partial t} \rho_{n m}(\epsilon, t)\right|_{\text {coll }}=-\gamma_{p}(\epsilon, t) \rho_{n m}(\epsilon, t),
$$

where $\epsilon$ is the excess energy with respect to the unrenor- malized band gap and the density- and energy-dependent damping rate is given by

$$
\gamma_{p}(\epsilon, t)=\gamma_{p}^{0}+c_{p} n^{1 / 3}(t)\left(1+\frac{\epsilon}{\Delta_{0}+a\left(\frac{\epsilon_{0}}{\epsilon}\right)^{3}}\right) .
$$

The damping (7) increases with excess energy $\epsilon$ because of the increasing number of decay channels. Parameters are as follows: $\gamma_{p}^{0}=1 /(300 \mathrm{fs}), c_{p}=3.4 \times$ $10^{-8} \mathrm{~cm}^{3} \mathrm{fs}^{-1}, \Delta_{0}=25 \mathrm{meV}, a=1 \mathrm{meV}$, and $\epsilon_{0}=$ $1400 \mathrm{meV}$. For the longitudinal relaxation, however, an energy-dependent rate would not be a conserving approximation. Hence, we use an energy-independent rate $\gamma_{e}(t)$ for the longitudinal $(e)$ relaxation

$$
\left.\frac{\partial}{\partial t} \rho_{n n}(\epsilon, t)\right|_{\text {coll }}=-\gamma_{e}(t)\left(\rho_{n n}(\epsilon, t)-f^{0}[\epsilon, n(t)]\right),
$$

where $f^{0}[\epsilon, n(t)]$ is the thermal $(T=300 \mathrm{~K})$ equilibrium Fermi function belonging to the density at time $t$ and

$$
\gamma_{e}(t)=\gamma_{e}^{0}+c_{e} n^{1 / 3}(t) .
$$

Parameters are $\gamma_{e}^{0}=1 /(160 \mathrm{fs})$ and $c_{e}=7.8 \times$ $10^{-8} \mathrm{~cm}^{3} \mathrm{fs}^{-1}$. Furthermore, we use an optical pulse $E(t)=\tilde{E}(t) \cos \left(\omega_{0} t+\phi\right)$ with Gaussian envelope $\tilde{E}(t)=\tilde{E}_{0} \exp \left[-2 \ln (2)\left(t^{2} / \delta t^{2}\right)\right]$, carrier photon energy $\hbar \omega_{0}=1.38 \mathrm{eV}$, carrier-envelope phase $\phi=0$ [17] and a full width at half maximum of the temporal intensity profile of $\delta t=7 \mathrm{fs}$. Finally, the third-harmonic spectra are proportional to $\left|P\left(\omega \approx 3 \omega_{0}\right)\right|^{2}$, where $P(\omega)$ is the Fourier transform of the total polarization $P(t)=\sum_{\vec{k}} d_{\vec{k}} \rho_{\mathrm{cv}, \vec{k}}(t)+$ c.c.

Theoretical results. - Two calculated third-harmonic spectra are shown in Fig. 2. For the lower field of $\tilde{E}_{0}=$ $0.23 \times 10^{9} \mathrm{~V} / \mathrm{m}$, the computed third-harmonic spectrum (lower dashed curve) consists of a single maximum, roughly centered around the third harmonic of the GaAs band edge, in agreement with experiment (lower solid curve). The theoretical peak is somewhat broader, which is likely due to the approximations made (pulse shape and/or scattering processes). For the higher field of $\tilde{E}_{0}=1.65 \times 10^{9} \mathrm{~V} / \mathrm{m}$, the theoretical spectrum (upper dashed curve) exhibits three maxima around 4.3, 3.8, and $3.5 \mathrm{eV}$, again in agreement with the experiment (upper solid curve). Because of their energetic separation we interpret these three peaks as manifestations of the two-band Mollow triplet. The splitting has to be calculated from the appropriately averaged renormalized Rabi frequency. The triplet is not symmetrical around $3 E_{\mathrm{g}}$ but is rather redshifted due to renormalization effects. Also note that the absolute value of the estimated "high" peak electric field of $\tilde{E}_{0}=2.1 \times 10^{9} \mathrm{~V} / \mathrm{m}$ inside the GaAs film is merely $21 \%$ larger than the corresponding value of $\tilde{E}_{0}=1.65 \times 10^{9} \mathrm{~V} / \mathrm{m}$ used in the calculations. This difference is well within the errors of the experimental estimate and the accuracy with which the theory is able to determine the correct dipole matrix element. Notice that the chosen pulse duration of $7 \mathrm{fs}$ is somewhat larger than the experimental duration of 5 fs (see above). This 


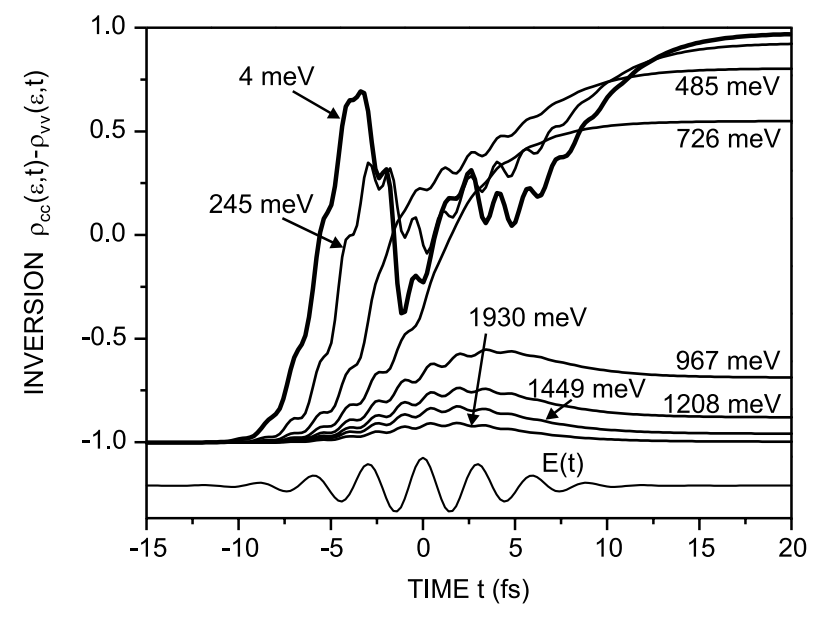

FIG. 3. Calculated inversion for various excess energies $\epsilon$ versus time $t$ for a peak electric field of $\tilde{E}_{0}=1.65 \times$ $10^{9} \mathrm{~V} / \mathrm{m}$. Parameters are identical to Fig. 2. For $t=20 \mathrm{fs}$ the carrier density equals $1.1 \times 10^{20} / \mathrm{cm}^{3}$. The lower part shows the laser electric field $E(t)$ versus time.

effectively accounts for remaining pulse distortions due to propagation effects in the GaAs film [12] and/or for some residual chirp on the incident pulses. For $\delta t=5 \mathrm{fs}$, the third-harmonic spectra tend to exhibit a shift towards higher energies in comparison with the experiment. The detailed shape of the third-harmonic spectra also depends on the shape of the pulse envelope $\tilde{E}(t)$. For the investigation of this aspect we have used a slightly widened "experimental pulse shape" and found (not shown) that one can further improve the agreement with experiment, at the cost of additional fitting parameters.

To further support our interpretation in terms of the two-band Mollow triplet in the frequency domain corresponding to Rabi flopping of band-to-band transitions in the time domain, we depict the inversion components, $\rho_{\mathrm{cc}}(\epsilon, t)-\rho_{\mathrm{vv}}(\epsilon, t)$, in Fig. 3 for various excess energies $\epsilon$. Parameters are identical to high excitation in Fig. 2. The structures with twice the frequency of light due to the carrier-wave oscillation lead to third-harmonic generation, their modulation to the triplet in Fig. 2. At small excess energies (close to the band edge), the inversion nearly performs a complete Rabi flop during the first half of the pulse $(t<0)$. A second Rabi flop roughly between $t=-1 \mathrm{fs}$ and $t=6 \mathrm{fs}$ is already very heavily damped. At later times, dissipative rather than coherent kinetics dominates and the low-energy states are filled from above. At large excess energies, where the dephasing is stronger, only an irreversible increase of the inversion is left after the pulse has passed. Based on our experience with quantum kinetics $[15,16]$, we expect that the window of coherent dynamics would be widened to a certain extent by the use of a non-Markovian retarded description of electron-electron scattering. We have checked that replacing the above electron-phonon scatter- ing by electron-phonon quantum kinetics leads to no significant changes.

In conclusion, we have observed a triplet peak structure in our experiments on thin films of the model semiconductor GaAs. Fully dynamical solutions of the semiconductor Bloch equations are in almost quantitative agreement with the experiment. This comparison allows us to assign the peak structure to the two-band Mollow triplet, which is an immediate consequence of the lightinduced gaps in the original conduction and valence band, respectively. A key parameter is the Rabi energy. Only if it becomes comparable to the photon energy, optical transitions between the induced bands have sufficient spectral weight throughout an appreciable fraction of momentum space. Finally, we note that we have also found the modulation due to the two-band Mollow triplet in calculations [18] in a pump/probe geometry for slightly negative delay times and for otherwise similar excitation conditions. This shows that light-induced gaps in semiconductor bands can possibly be observed also by other experimental techniques.

We thank L. Bányai and P. Gartner for their contributions to the formulation of semiconductor Bloch equations with full bands. We also thank U. Morgner and F. X. Kärtner for help with setting up the laser as well as for stimulating discussions. The group of H. H. acknowledges the support by the DFG (Ha 1197/15-1) and the use of computing facilities at the Frankfurt Center for Scientific Computing. The work of M.W. is supported by Projects No. DFG-We 1497/11-1 and No. DFG-We 1497/9-1. H. M. G. and G. K. thank NSF AMOP for support.

[1] H. Haug, in Optical Nonlinearities and Instabilities, edited by H. Haug (Academic, New York, 1988), p. 53.

[2] V. F. Elesin, Sov. Phys. JETP 32, 328 (1971).

[3] C. Comte and G. Mahler, Phys. Rev. B 34, 7164 (1986).

[4] S. Schmitt-Rink et al., Phys. Rev. B 37, 941 (1988).

[5] F. Jahnke and K. Henneberger, Phys. Rev. B 45, 4077 (1992).

[6] F. Y. Wu et al., Phys. Rev. Lett. 38, 1077 (1977).

[7] C. Cohen-Tannoudji, J. Dupont-Roc, and G. Grynberg, Atom-Photon Interactions (Wiley, New York, 1992).

[8] J.-P. Likforman et al., Phys. Rev. Lett. 79, 3716 (1997).

[9] F. Quochi et al., Phys. Rev. Lett. 80, 4733 (1998).

[10] S. Hughes, Phys. Rev. Lett. 81, 3363 (1998).

[11] O. D. Mücke et al., Phys. Rev. Lett. 87, 057401 (2001).

[12] O. D. Mücke et al., Phys. Rev. Lett. 89, 127401 (2002).

[13] U. Morgner et al., Opt. Lett. 24, 411 (1999).

[14] The $\vec{p} \cdot \vec{A}$ coupling delivers qualitatively similar results.

[15] Q. T. Vu et al., Phys. Rev. Lett. 85, 3508 (2000).

[16] W. A. Hügel et al., Phys. Rev. Lett. 83, 3313 (1999).

[17] In the theory, the third-harmonic spectrum for $\phi=0$ is practically identical to the $\phi$-averaged spectrum, which is measured in the experiment.

[18] Q. T. Vu and H. Haug (unpublished). 